Research Paper

\title{
Increasing the Inflammatory Competence of Macro- phages with IL- 6 or with Combination of IL-4 and LPS Restrains the Invasiveness of Pancreatic Cancer Cells
}

\author{
Aino N.E. Salmiheimoㄹ, Harri K. Mustonen1, Sanna A.A. Vainionpää1, Zhanlong Shen², Esko A.J. \\ Kemppainen ${ }^{1}$, Hanna E. Seppänen ${ }^{1 *}$, Pauli A. Puolakkainen ${ }^{1 * 凶}$ \\ 1. Department of Surgery, Helsinki University Hospital and University of Helsinki, Helsinki, Finland \\ 2. Department of Gastroenterological Surgery, Peking University People's Hospital, Beijing, China \\ *H. Seppänen and P. Puolakkainen share equal last authorship. \\ $\square$ Corresponding author: Pauli Antero Puolakkainen, MD, PhD, Professor of Surgery. Address: Department of Surgery, Helsinki University Central Hospital, \\ Kasarmikatu 11-13, P.O. Box 263, 00130 Helsinki, FINLAND. Phone number: +358 50582 9785; E-mail: pauli.puolakkainen@hus.fi
}

(๑) Ivyspring International Publisher. Reproduction is permitted for personal, noncommercial use, provided that the article is in whole, unmodified, and properly cited. See http://ivyspring.com/terms for terms and conditions.

Received: 2015.06.10; Accepted: 2015.09.07; Published: 2016.01.01

\begin{abstract}
Recent studies suggest that pro-inflammatory type MI macrophages inhibit tumor progression and that anti-inflammatory M2 macrophages enhance it. The aim of this study was to examine the interaction of type $M 1$ and $M 2$ macrophages with pancreatic cancer cells. We studied the migration rate of fluorescein stained pancreatic cancer cells on Matrigel cultured alone or with Granulocyte-Macrophage Colony Stimulating Factor (GM-CSF) differentiated macrophages or with Macrophage Colony Stimulating Factor (M-CSF) differentiated macrophages, skewing the phenotype towards pro- and anti-inflammatory direction, respectively. Macrophage differentiation was assessed with flow cytometry and the cytokine secretion in cell cultures with cytokine array. Both GM-CSF and M-CSF differentiated macrophages increased the migration rate of primary pancreatic adenocarcinoma cell line (MiaPaCa-2) and metastatic cell line (HPAF-II). Stimulation with IL6 or IL4+LPS reversed the macrophages' increasing effect on the migration rate of MiaPaCa-2 completely and partly of HPAF-II. Co-culture with MiaPaCa-2 reduced the inflammatory cytokine secretion of GM-CSF differentiated macrophages. Co-culture of macrophages with pancreatic cancer cells seem to change the inflammatory cytokine profile of GM-CSF differentiated macrophages and this might explain why also GM-CSF differentiated macrophages promoted the invasion. Adding IL6 or IL4+LPS to the cell culture with MiaPaCa-2 and GM-CSF or M-CSF differentiated macrophages increased the secretion of inflammatory cytokines and this could contribute to the reversion of the macrophage induced increase of cancer cell migration rate.
\end{abstract}

Key words: pancreatic adenocarcinoma, M1, M2, macrophages, invasion

\section{Introduction}

Ductal adenocarcinoma is the most common malignancy of the pancreas and worldwide pancreatic cancer is among the deadliest forms of cancer. It typically metastases aggressively at early stages and has an overall 5-year survival rate of only $8 \%$ [1]. The preliminary step for the process of metastasis is usually invasion through the extracellular matrix (ECM). Chronic inflammation, such as in chronic pancreatitis, significantly increases the risk of cancer $[2,3]$ and lately the relationship between inflammation and cancer has been a subject of great interest.

Monocytes are among the first cells to infiltrate a damaged tissue where they differentiate into mature macrophages. In response to cytokines and other stimuli of their micro-environment macrophages polarize towards either pro-inflammatory type 1 (M1) or anti-inflammatory type 2 (M2) macrophages [4,5]. M1 macrophages are cytotoxic, they stimulate the im- 
mune system and inhibit tumor progression. Their phenotype is generally characterized by high interleukin (IL) 12 and IL23 and low IL10. M2 macrophages promote tissue remodeling and repair and activate angiogenesis. They enhance wound healing by suppressing the immune system. M2 macrophages are characterized by a low IL12 and IL23 and high IL10 phenotype [6,7]. This distinction is a simplified view of the two extremes of the continuum of macrophage polarization and due to their plasticity even mature macrophages can alter their polarization.

In the presence of cancer cells macrophages differentiate into tumor associated macrophages (TAMs) which is a distinct subpopulation of M2 polarized macrophages [6]. In various forms of cancers, including the pancreatic cancer, the presence of TAMs is associated with a worse prognosis [8]. TAMs increase the invasion of pancreatic cancer cells in cell cultures and in mouse models $[9,10]$. They facilitate cancer invasion e.g. by producing mediators that degrade the ECM, thus, allowing tumor growth and enabling the tumor cells to migrate through tissue barriers $[9,11]$.

In in vitro -studies Granulocyte-Macrophage Colony Stimulating Factor (GM-CSF) and Macrophage Colony Stimulating Factor (M-CSF) have been used to polarize maturing monocytes towards type M1 and M2 macrophages, respectively [7,12,13]. Different additional stimuli are used to skew the polarization towards different subtypes of M1 and M2 macrophages [14]. IL6 is an inflammatory cytokine released by pro-inflammatory M1 macrophages [15]. It activates STAT-3 pathway that is associated with inflammation-induced tumorigenesis [16]. Lipopolysaccharide (LPS) activates the classical way via toll-like receptor that skews the macrophage polarization towards M1 and increases for example the expression of Tumor Necrosis Factor alpha (TNFa) in macrophages [17]. T-helper (Th) 2 cytokine IL4 activates monocytes to polarize towards the alternative M2 macrophages [18].

The aim of this study was to examine the relation of pro- and anti-inflammatory macrophages with the invasiveness of pancreatic cancer. We wanted to find ways to interfere with pancreatic cancer cell migration through macrophage polarization. The hypothesis was that GM-CSF skews the macrophage polarization towards M1 and M-CSF towards M2 macrophages. To explore the macrophage plasticity we intended to stimulate GM-CSF differentiated macrophages further towards pro-inflammatory phenotype by adding IL6 to the cell culture and to generate a mixed phenotype between M1 and M2 to M-CSF differentiated macrophages by adding anti-inflammatory IL4 and pro-inflammatory LPS. We explored the changes in pancreatic cancer cell migration, intercellular cytokine signaling and in the macrophages' surface expression. The aim was to change the macrophage polarization after their maturation to assess their potential in inhibition of pancreatic cancer migration.

\section{Materials and methods}

\section{Cell cultures and reagents}

Human mononuclear cells were isolated from five different healthy subjects' blood samples by density gradient centrifugation with Ficoll-Paque Plus (Amershamn, Uppsala, Sweden). This was followed by paramagnetic bead separation with Human Monocyte Isolation Kit II (Miltenyi Biotec, Auburn, USA) according to the manufacturer's instructions. Blood samples from different individuals were processed and used in assays separately. Macrophages from the same donor were used as internal controls in each of the study settings.

All cells were cultured in Macrophage Serum-free Media (Gibco Life Technologies, Paislay, UK) supplemented with penicillin (Sigma, St.Louis, USA) $100 \mathrm{mg} / \mathrm{ml}$. To differentiate the isolated monocytes into mature macrophages we added GM-CSF (ImmunoTools, Oldenburg, Germany) $10 \mathrm{ng} / \mathrm{ml}$ or M-CSF (ImmunoTools) $50 \mathrm{ng} / \mathrm{ml}$ to the culture media and incubated them in standard $37{ }^{\circ} \mathrm{C}$ and $5 \% \mathrm{CO} 2$ for 5 days. The additional stimuli, LPS $10 \mathrm{ng} / \mathrm{ml}$ (Sigma) and IL4 $20 \mathrm{ng} / \mathrm{ml}$ (R\&D Systems, Shanghai, China) or IL6 $50 \mathrm{ng} / \mathrm{ml}$ (R\&D Systems), were added after 5 days of monocyte differentiation.

The human pancreatic adenocarcinoma cell lines MiaPaCa-2 (primary tumor cell line) and HPAF-II (metastatic tumor cell line) were purchased from the American Type Culture Collection. MiaPaCa-2 and HPAF-II were either cultured alone using the same media as for the macrophages or added to the macrophage cultures after 5 days of monocyte isolation and differentiation. The study has been approved by Ethics Committee of Helsinki University Central Hospital.

\section{Macrophage characterization}

To assess the surface expression of the differentiated macrophages they were cultured on Nunc UpCell dishes (Thermo Scientific). First, the isolated monocytes were differentiated into macrophages with GM-CSF or M-CSF for 5 days. Then the additional stimuli IL4 + LPS/IL6 and/or MiaPaCa-2 cells or neither were added and the cells were cultured for further 48 hours. The cells were detached from the UpCell dishes according to the manufacturer's instructions by incubating the cells in room temperature for 40 minutes. After detaching the cells, macrophages were separated from the cancer cells with anti-CD11b magnetic micro beads (Miltenyi Biotec Inc., Auburn, 
USA) and labeled with selected antibodies for 20min in room temperature. They were acquired on FACS Calibur (CellQuest Pro software; BD Bioscience) flow cytometer and the data was analyzed with WinMDI software (v2.8). The antibodies we used were Mouse Anti-Human FITC and PE CD14, FITC CD16, PE CD80, APC CD86, PE CD163, APC CD206, PE CD209, PE Mouse IgG1 $\mathrm{\kappa}$ Isotype Control, APC Mouse IgG1 к Isotype Control, FITC Mouse IgG1 k Isotype Control (BD Pharmingen, San Diego, USA).

\section{Cancer cell migration study}

Cells were cultured on 8-well coverslip dishes (Nunc, Thermo Scientific, Rochester, USA) coated with $60 \mu 1$ Matrigel (BD Biosciences, San Jose, USA). As described, the monocytes were first differentiated into macrophages with GM-CSF or M-CSF on Matrigel for 5 days, after which the additional stimuli IL4+LPS or IL6 were added. Simultaneously was added MiaPaCa-2 and HPAF-II cells, which were stained with fluorescent dye (CellTracker Green CMFDA, Invitrogen, Eugene, USA). We let the cells settle in a standard cell incubator for 24 hours before the fluorescence microscopy.

The cancer cell migration on Matrigel was assessed by recording their movement with $30 \mathrm{~min}$ intervals for 24 hours in fluorescence microscope equipped with coolled CCD camera (Sensicam, PCO, Germany) and humidified, temperature $\left(+37^{\circ} \mathrm{C}\right)$ and $\mathrm{CO}_{2}(5 \%)$ controlled chamber (OKOlab, Ottaviano, Italy), as previously described [19]. The data was analyzed using ImagePro software (v 7.01, Media Cybernetics, Rockville, MD, USA). The stained cancer cells were observed with and without the presence of the differentiated macrophages and the stimuli IL4 + LPS or IL6.

\section{Cytokine array}

For the cytokine array the monocytes were first differentiated into macrophages for 5 days with culture media supplemented with either GM-CSF or M-CSF. At this point, the media was refreshed in all cell cultures and the additional stimuli (IL4 + LPS for M-CSF stimulated cells and IL6 for GM-CSF stimulated cells) and/or MiaPaCa-2 cells were added to the cell cultures. After 24 hours of incubation in standard $37{ }^{\circ} \mathrm{C}$ and $5 \% \mathrm{CO} 2$ incubator, the cell culture media was collected. The collected medium was concentrated from $1.5 \mathrm{ml}$ by $3 \mathrm{~K}$ filters (Amicon Ultra Centrifugal Units, Millipore Corporation, Billerica, MA, USA). The concentrated medium was incubated with pre-coated Human Cytokine Array Panel A (Proteome Profiler Arrays, R\&D Systems) according to the manufacturer's instructions. The arrays were imaged with the Odyssey infrared imager (Licor Biosciences,
Lincoln, USA). The dot blots were analyzed densitometrically with Odyssey software (Licor Biosciences).

\section{Statistics}

To detect the difference between continuous variables we used the nonparametric Mann-Whitney U test or Wilcoxon's signed-rank test (paired measurements). $\mathrm{p}$ values $<0.05$ were considered statistically significant. The results are presented as mean \pm standard error of mean (SEM). In the migration assay confidence intervals (CI) for fold change were calculated according to the rules of error propagation as asymptotic confidence intervals. Cytokine array results are given as percentage between negative and positive control recorded on each array.

\section{Results}

\section{Macrophage characteristics}

We used several surface markers (M1 markers: CD16, CD80, CD86 and M2 markers: CD14, CD163, CD206, CD209) to characterize the differentiated macrophages using flow cytometry (Fig. 1). When comparing GM-CSF and M-CSF differentiated macrophages the proportion of macrophages positive to M2 markers CD209 ( $p=0.004), C D 163(p=0.001)$, and CD14 ( $p=0.007$ ) was greater in M-CSF differentiated macrophages but M2 marker CD206 was conversely less positive $(p=0.001)$. Of M1 markers the only significant difference was that the proportion of macrophages positive to CD80 was greater in M-CSF differentiated macrophages $(p=0.017)$. These findings show that GM-CSF differentiated macrophages are only slightly more polarized towards M1 (as the majority of M2 markers are lower in these macrophages) and M-CSF differentiated macrophages towards M2 but they also show that the division isn't unambiguous. Co-culturing MiaPaCa-2 with GM-CSF differentiated macrophages increased the CD16 positivity of the macrophages $(p=0.002)$. MiaPaCa-2 cells did not induce significant changes in M-CSF differentiated macrophages' surface expression.

Adding IL4 + LPS to M-CSF differentiated macrophages increased the expression of M2 markers CD209 $(p=0.001)$ and CD206 $(p=0.005)$ whereas the expression of M2 markers CD163 $(p=0.001)$ and CD14 $(p=0.007)$ decreased and M1 marker CD16 increased $(\mathrm{p}=0.022)$ (Fig. 1). When MiaPaCa-2 cells were added to M-CSF + IL4 + LPS differentiated macrophages the increased proportion of cells positive to CD206 $(p=0.008)$ and decreased CD163 $(p=0.008)$ and CD14 $(\mathrm{p}=0.004)$ positivity. Adding IL6 induced no statistically significant differences to the surface expression of GM-CSF differentiated macrophages with nor without MiaPaCa-2. 


\section{IL6 and IL4 + LPS reversed the macro- phage-induced increase of pancreatic cancer cell migration}

Both GM-CSF and M-CSF differentiated macrophages increased the migration rate of pancreatic cancer cells in Matrigel. GM-CSF differentiated macrophages increased the migration rate of primary pancreatic adenocarcinoma tumor cell line (Mi$\mathrm{aPaCa}-2$ ) from $11.4 \mu \mathrm{m} / \mathrm{h} \pm 1.0$ to $24.1 \mu \mathrm{m} / \mathrm{h} \pm 1.7$ $(\mathrm{p}<0.001$, increase 2.1 fold (95\% CI 1.6-2.6)) and metastatic cell line (HPAF-II) from $4.7 \mu \mathrm{m} / \mathrm{h} \pm 0.4$ to
$18.4 \mu \mathrm{m} / \mathrm{h} \pm 2.5(\mathrm{p}<0.001$, increase 3.9 fold $(95 \% \mathrm{CI}$ 2.7-5.1)). M-CSF differentiated macrophages increased the migration rate of MiaPaCa-2 cells from $11.4 \mu \mathrm{m} / \mathrm{h}$ \pm 1.1 to $16.7 \mu \mathrm{m} / \mathrm{h} \pm 1.6(\mathrm{p}=0.011$, increase 1.5 fold (95\% CI 1.1-1.9)) and of HPAF-II from $5.5 \mu \mathrm{m} / \mathrm{h} \pm 0.8$ to $20.9 \mu \mathrm{m} / \mathrm{h} \pm 1.4(\mathrm{p}<0.001$, increase 3.8 fold $(95 \% \mathrm{CI}$ 2.6-5.0)). GM-CSF differentiated macrophages increased the migration rate of MiaPaCa-2 cells unexpectedly slightly more than M-CSF differentiated macrophages ( $p=0.007)$. (Fig. 2)

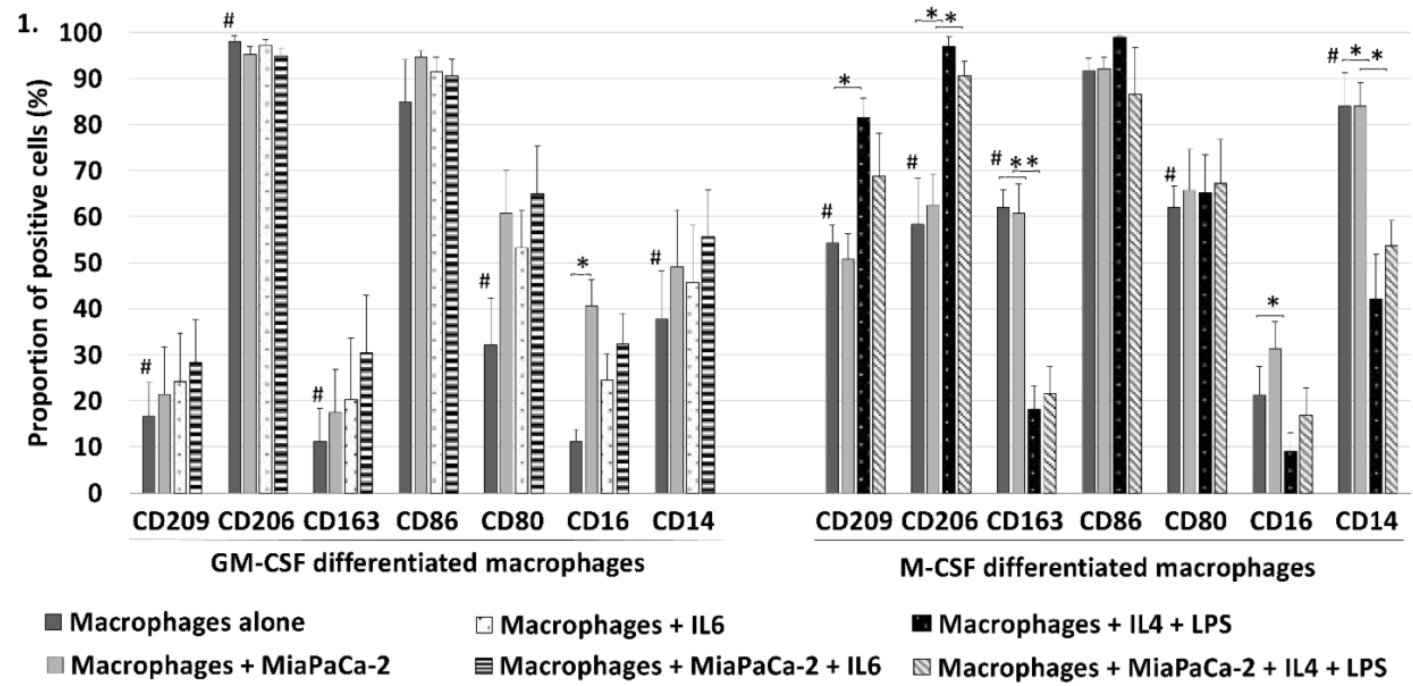

Figure 1. GM-CSF (skewed towards M1) and M-CSF (skewed towards M2) differentiated macrophages expressed different surface protein distribution in flow cytometry. Comparing GM-CSF and M-CSF macrophages proportion of cells positive to M2 markers CD209, CD163 and CD14 was significantly higher in M-CSF differentiated macrophages but M2 marker CD206 was less positive and also M1 marker CD80 was higher (\#p<0.05). This shows a partly incomplete polarization with these commonly used stimulants. MiaPaCa-2 cells increased only the CD16 positivity of GM-CSF macrophages but had no significant effect on M-CSF differentiated macrophages. IL6 induced no significant changes to the surface expression of GM-CSF differentiated macrophages. Adding IL4 and LPS to M-CSF differentiated macrophages increased the proportion of cells positive to M2 markers CD209 and CD206 and to M1 marker CD16 but M2 markers CD163 and CD14 decreased (*p<0.05). Error bars show the standard error of mean.

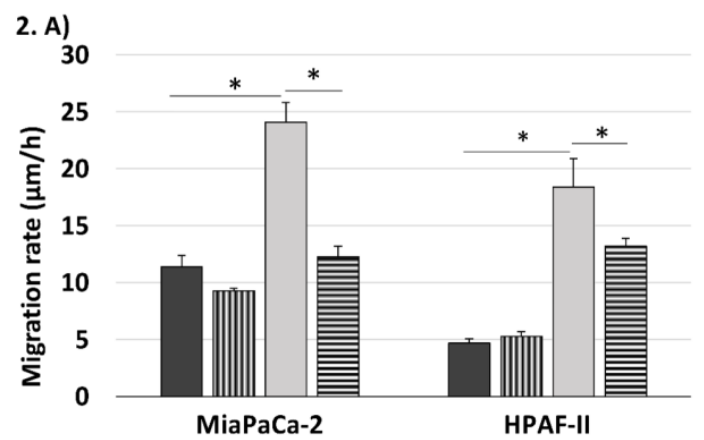

2. B)

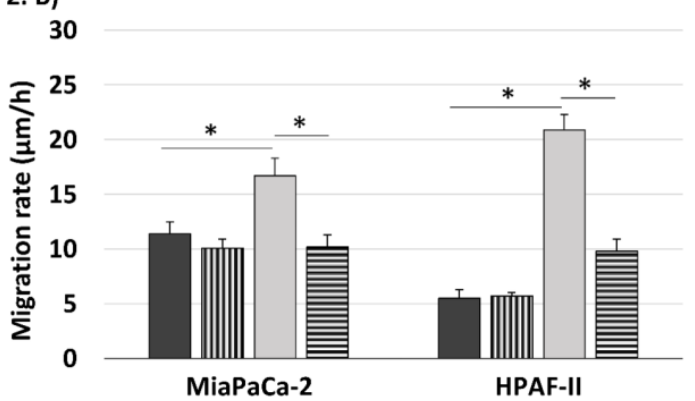

Cancer cells alone with GM-CSF

moncer cells alone with GM-CSF + IL6

$\square$ Macrophages and cancer cells with GM-CSF

目 Macrophages and cancer cells with GM-CSF + IL6

Cancer cells alone with
M-CSF
III Cancer cells alone with
M-CSF + IL4 + LPS
Macrophages and cancer
cells with M-CSF
Macrophages and cancer
cells with M-CSF + IL4 +
LPS

Figure 2. Both GM-CSF and M-CSF differentiated macrophages increased the migration rate of pancreatic cancer cells. This could be reversed by adding IL6 to GM-CSF or IL4 and LPS to M-CSF differentiated cell culture. MiaPaCa-2 and HPAF-Il cell invasion rate was measured in Matrigel cultured alone and with macrophages when they were cultured in medium containing (A) GM-CSF with and without IL6 and (B) M-CSF with and without IL4 and LPS. Error bars show the standard error of mean. * $<<0.05$. 
The additional stimuli IL6 and IL4 + LPS decreased the macrophage-induced invasiveness of pancreatic adenocarcinoma cells. When the cancer cells and macrophages were differentiated with GM-CSF + IL6 the migration rate of MiaPaCa-2 cells was $12.3 \mu \mathrm{m} / \mathrm{h} \pm 0.9$ (2.0 fold decrease compared to MiaPaCa-2 cells co-cultured with macrophages differentiated with only GM-CSF (95\% CI 1.6-2.4), $\mathrm{p}<0.001$ ) and of HPAF-II cells it was $13.2 \mu \mathrm{m} / \mathrm{h} \pm 0.7$ (1.4 fold decrease compared to HPAF-II cells co-cultured with macrophages differentiated with GM-CSF (95\% CI 1.0-1.8), $\mathrm{p}=0.044)$. The migration rate of MiaPaCa-2 cells co-cultured with M-CSF + IL4 + LPS differentiated macrophages was $10.2 \mu \mathrm{m} / \mathrm{h} \pm 1.1$ (1.6 fold decrease compared to MiaPaCa-2 cells and macrophages differentiated with M-CSF $(95 \%$ CI 1.2-2.1), $\mathrm{p}=0.003$ ) and of HPAF-II cells it was $9.8 \mu \mathrm{m} / \mathrm{h}$ \pm 1.1 (2.1 fold decrease compared to HPAF-II cells and macrophages differentiated with M-CSF (95\% CI 1.6-2.7), $\mathrm{p}=0.007)$. The additional stimuli IL6 and IL4 + LPS induced no significant changes to the migration rate of the pancreatic cancer cells cultured without macrophages.

\section{IL6 and IL4 + LPS increased the inflammatory cytokine response in co-cultures of pancreatic cancer cells and macrophages}

The cytokine panel searched the cell culture medium for numerous different cytokines and here we discuss only the statistically significant changes that we found. GM-CSF differentiated macrophages co-cultured with MiaPaCa-2 cells released less inflammatory cytokines TNFa $(p=0.018)$ and IL23 $(p=0.005)$ than the GM-CSF differentiated macrophages cultured alone and simultaneously decreased the secretion of anti-inflammatory IL13 $(p=0.010)$ and IL-1Ra (interleukin 1 receptor antagonist, $\mathrm{p}<0.001$ ). Adding IL6 to GM-CSF differentiated cell culture with macrophages and MiaPaCa-2 cells increased the expression of inflammatory cytokines TNFa $(\mathrm{p}=0.001)$, IL23 ( $p=0.004)$, and CCL1 ( $p=0.033)$. (Fig. 3. a)
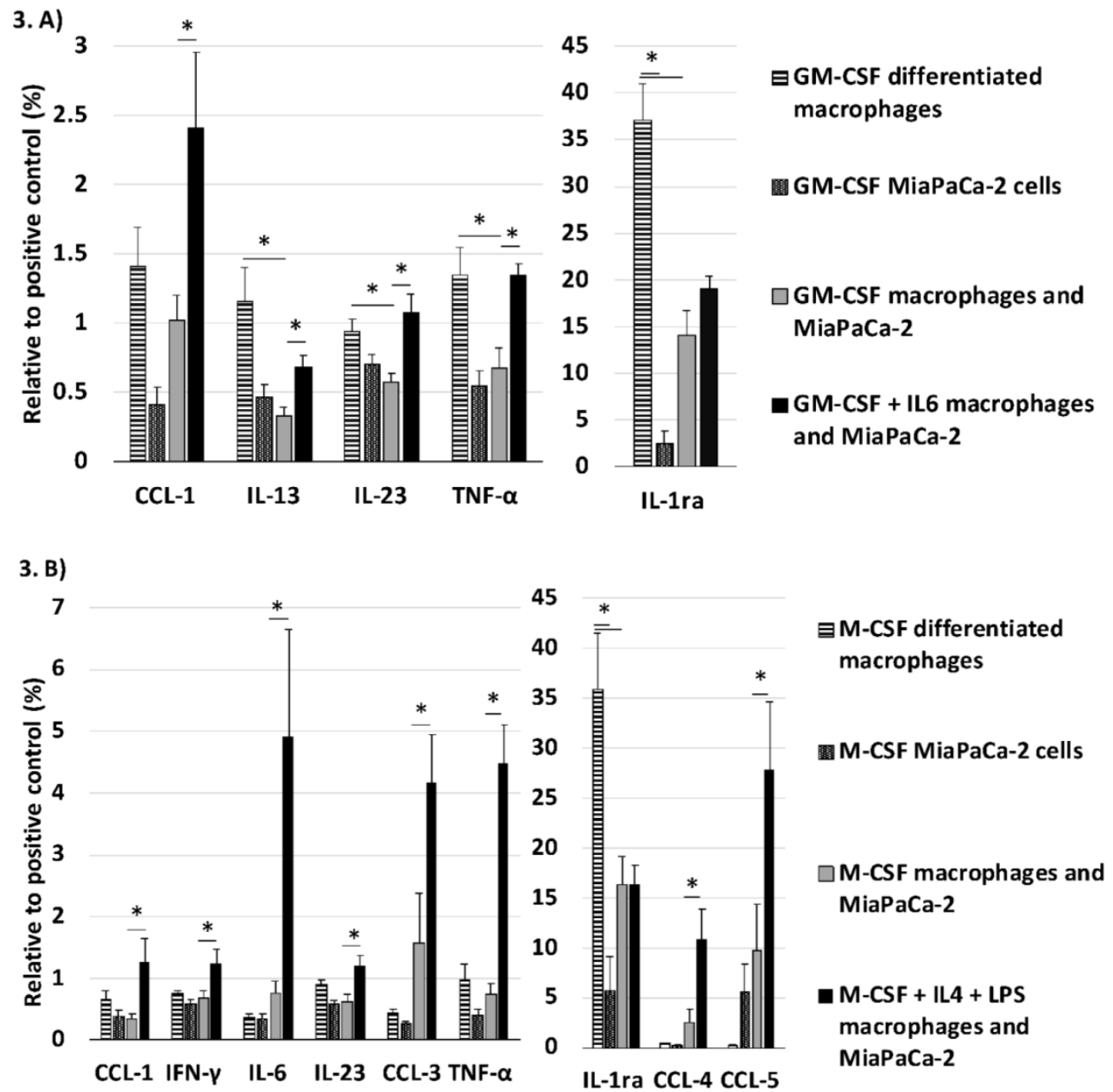

Figure 3. Cytokine secretion of macrophages and pancreatic cancer cells was studied with cytokine array. These figures show only the cytokines where significant changes observed on the array panel. (A) When GM-CSF differentiated macrophages were co-cultured with pancreatic cancer cells their inflammatory cytokine secretion decreased significantly. When IL6 was added to the cell culture the inflammatory cytokine secretion was restored. (B) In M-CSF differentiated macrophages co-cultured with pancreatic cancer cells stimulation with IL4 and LPS activated the secretion of inflammatory cytokines. Error bars show the standard error of mean. * $\mathrm{P}<0.05$ 
Co-culture with MiaPaCa-2 cells decreased the secretion of IL-1Ra of M-CSF differentiated macrophages $(p=0.007)$. Adding IL4+LPS to the cell culture with M-CSF differentiated macrophages and MiaPaCa-2 cells increased the expression of IL6 $(p=0.033)$, CCL1 $(p=0.035)$, CCL3 $(p=0.042), C C L 4$ $(p=0.027), C C L 5(p=0.045), I N F Y(p=0.045)$, and TNFa $(\mathrm{p}<0.001)$. (Fig. 3. b)

\section{Discussion}

Inflammation and the cells associated with it play a critical role in formation and progression of cancer. Our study endorses the fact that macrophages have a drastic impact on pancreatic cancer cell migration and that their effect can be influenced even after their maturation. This study demonstrated that in Matrigel, simulating the in vivo extra cellular matrix environment, the migration of pancreatic cancer cells increased in the presence of both GM-CSF differentiated and M-CSF differentiated macrophages. By adding IL6 to GM-CSF differentiated and IL4 + LPS to M-CSF differentiated co-cultures we were able to reduce the macrophage-induced increase in pancreatic cancer cell migration and increase the overall inflammatory cytokine profile in the co-culture.

In previous studies GM-CSF differentiated macrophages have displayed superior ability in suppressing tumor proliferation as compared to M-CSF differentiated macrophages [14]. Our study questions the assumption that GM-CSF differentiated macrophages solely inhibit tumor progression as they increased the migration rate of pancreatic cancer cells. It was evident that in our study the macrophages' cell surface expression was not completely skewed to the extremes of macrophage polarization as some M2 markers were higher in GM-CSF differentiated macrophages than in M-CSF differentiated macrophages and vice versa. Furthermore, the pancreatic cancer cells and the additional stimuli induced surprisingly little change to the macrophages' surface protein expression. This might result from the study setting as GM-CSF and M-CSF were present in the macrophage culture from the beginning of monocyte maturation and the additional stimuli and pancreatic cancer cells were added later. This suggests also that the distinction of macrophages to type M1 and type M2 according to their surface protein expression can be unreliable in predicting their effect on the pancreatic cancer migration, as the additional stimuli didn't change the macrophage polarization according to their surface expression but still their influence to the pancreatic cancer cell migration was altered. Also in some previous studies TAMs have shown ability to promote tumor invasion regardless of their phenotypic polarization to M1 macrophages with GM-CSF [20,21].
A possible explanation for the enhancement of pancreatic cancer cell migration rate with GM-CSF differentiated macrophages was revealed in cytokine array: pancreatic cancer cells were able to reduce the GM-CSF differentiated macrophages' inflammatory cytokine secretion while the surface expression of these macrophages remained substantially intact. Previous studies have shown that due to their plasticity mature macrophages change their cytokine secretion in response to interaction with pancreatic cancer cells [22]. When the cells were differentiated with a combination of GM-CSF + IL6 or M-CSF + IL4 + LPS the macrophages' increasing effect on the migration rate of primary pancreatic cancer cell line was almost completely reversed and significantly reduced in the metastatic cell line. Cytokine array illustrated that the additional stimuli elevated the inflammatory cytokine and chemokine secretion in the co-culture. In this study IL6 with GM-CSF and the combination of anti-inflammatory IL4 and pro-inflammatory LPS with M-CSF appeared to skew the macrophage function towards pro-inflammatory and anti-tumor direction.

In both GM-CSF and M-CSF differentiated macrophages the secretion of interleukin 1 receptor antagonist (IL-1Ra) was significantly reduced when co-cultured with pancreatic cancer cells, suggesting an inflammatory response towards pancreatic cancer cells. IL-1Ra binds to IL1 receptor without activating it. IL1 $\alpha$ and IL1 $\beta$ are inflammatory cytokines that have been associated with metastasis worse prognosis in many types of cancer [23]. Interestingly, in our study the secretion of IL1 $\alpha$ and IL1 $\beta$ remained low in all cell cultures (data not shown). Of the cytokines observed TNFa and IL23 were elevated by the additional stimuli in both GM-CSF and M-CSF differentiated co-cultures. The weakness of this setting is obviously that we cannot determine if the increased cytokine secretion originates from macrophages or pancreatic cancer cells. TNFa is a cytokine that participates in inflammation, immunity, stimulation of growth factor synthesis, and apoptosis. It conducts a delicate balance between cytotoxicity and growth promotion and produces both pro- and anti-tumor effects [24]. In pancreatic cancer the clinical use of single TNFa treatment does not improve prognosis [25]. IL23 is one of the inflammatory cytokines characteristically secreted by pro-inflammatory M1 macrophages [6,7]. It is pivotal in autoimmune and chronic inflammation [26]. Its role in tumor progression is controversial but studies suggest that it is associated with increased cancer metastasis [27].

In the M-CSF differentiated co-cultures the addition of IL4 + LPS increased additionally the secretion of inflammatory IL6, C-C chemokine ligand (CCL) 1, CCL3, CCL4, CCL5, and INFY. The role of 
CCL1, CCL5 (also known as Regulated upon Activation, Normal T-cell Expressed, and Secreted, RANTES), a target gene of NF- $\kappa$ B, and CCL3 (also known as Macrophage Inflammatory Protein 1 alpha, MIP-1a), CCL4 (MIP-1 $\beta$ ) is still unclear in pancreatic cancer progression. They are in central role in inducing anti-tumorigenic immune responses but they are also associated with progression and metastasis in some types of cancer $[28,29]$.

Due to the complexity of the interactions of stromal elements in cancer surroundings in vivo these cytokines could have different effects on pancreatic cancer migration when exposed to other cells involved in inflammatory interactions, for example, the tumor-promoting actions of IL23 via Th-17 cells [30]. We do not suggest that these individual inflammatory cytokines by themselves inhibit the migration of pancreatic cancer cells but our study demonstrates the overall enhancement of the inflammatory status of macrophages and their microenvironment seem to inhibit pancreatic cancer cell migration in Matrigel. This needs further investigation in future studies.

Both GM-CSF and M-CSF differentiated macrophages increased the migration rate of metastatic pancreatic cancer cell line markedly more than of the primary tumor cell line. Further, with the metastatic cell line the macrophages' increasing effect on the migration rate was only partly reversed by the combination of GM-CSF + IL6 or M-CSF + IL4 + LPS. The metastatic cell line seems to have developed mechanisms to take more advantage of TAMs' tumor promoting abilities and ways to resist the inflammatory response. Previous studies have shown similar observations; hypoxia does not inhibit macrophage-induced migration in metastatic pancreatic cancer cells as it does in primary tumor cells and the expression of for example ADAM8, TIMP3, Vasohibin-1, VEGF-A, and VEGF-C is different in metastatic and primary tumor cell lines [9,31]. However, as we used only one primary and one metastatic cell line our results need to be confirmed in future studies.

As this is an in vitro study it is impossible to draw direct conclusions to in vivo situation. It would be beneficial to study the relevance of these findings in enhancing the inflammatory status of macrophages in in vivo models. Since macrophages participate essentially in pancreatic cancer invasion and the cancer cells are able to influence macrophage polarization and cytokine expression even after their maturation it is crucial to find ways to enhance the inflammatory competence of macrophages and further study their utility as potential targets for therapy.

\section{Conclusion}

Macrophages participate indisputably to pancreatic cancer cell invasion and understanding these interactions is essential for the development of pancreatic cancer treatment. Pancreatic cancer cells are able to inhibit inflammatory cytokine expression in GM-CSF differentiated macrophages. This explains why both GM-CSF (skewed towards pro-inflammatory M1) and M-CSF (skewed towards anti-inflammatory M2) differentiated macrophages promoted the invasion of pancreatic cancer cells. IL6 and IL4+LPS activated the inflammatory cytokine expression in the cell cultures and this could contribute to the reversion of the macrophage induced increase in cancer cell migration rate. Further studies are needed to apply these findings to the in vivo situation.

\section{Abbreviations}

ECM: extra cellular matrix; IL: interleukin; TAM: tumor associated macrophage; GM-CSF: Granulocyte-Macrophage Colony Stimulating Factor; M-CSF: Macrophage Colony Stimulating Factor; LPS: lipopolysaccharide, TNFa: Tumor Necrosis Factor alpha; CCL: C-C chemokine ligand

\section{Acknowledgements}

This study was supported by Sigrid Juselius Foundation and Helsinki University Central Hospital Research Funds. AS received a grant also from Biomedicum Helsinki Foundation and Finnish Medical Foundation.

\section{Competing Interests}

The authors have declared that no competing interest exists.

\section{References}

1. [Internet] National Cancer Institute, SEER Cancer Statistics Review, 1975-2012. http://seer.cancer.gov/csr/1975_2012/browse_csr.php?sectionSEL=22\&page SEL=sect_22_table.08.html.

2. Coussens LM, Werb Z. Inflammation and cancer. Nature. 2002; 420: 860-867.

3. Ekbom A, McLaughlin JK, Nyren O. Pancreatitis and the risk of pancreatic cancer. N Engl J Med. 1993; 329: 1502-1503.

4. Zhou D, Huang C, Lin Z, Zhan S, Kong L, Fang C, et al. Macrophage polarization and function with emphasis on the evolving roles of coordinated regulation of cellular signaling pathways. Cell Signal. 2014; 26: 192-197. doi: 10.1016/j.cellsig.2013.11.004

5. Mosser DM, Edwards JP. Exploring the full spectrum of macrophage activation. Nature Rev Immunol. 2008; 8: 958-969. doi: 10.1038/nri2448.

6. Allavena P, Sica A, Solinas G, Porta C, Mantovani A. The inflammatory micro-environment in tumor progression: the role of tumor-associated macrophages. Crit Rev Oncol Hematol. 2008; 66: 1-9.

7. Verreck FA, de Boer T, Langenberg DM, Hoeve MA, Kramer M, Vaisberg E, et al. Human IL-23-producing type 1 macrophages promote but IL-10-producing type 2 macrophages subvert immunity to (myco)bacteria. Proc Natl Acad Sci US A. 2004; 101: 4560-4565.

8. Kurahara H, Shinchi H, Mataki Y, Maemura K, Noma H, Kubo F, et al. Significance of M2-polarized tumor-associated macrophage in pancreatic cancer. J Surg Res. 2011; 167: e211-9. doi: 10.1016/j.jss.2009.05.026.

9. Puolakkainen P, Koski A, Vainionpaa S, Shen Z, Repo H, Kemppainen E, et al. Anti-inflammatory macrophages activate invasion in pancreatic adenocarci- 
noma by increasing the MMP9 and ADAM8 expression. Med Oncol. 2014; 31: 884. doi: 10.1007/s12032-014-0884-9.

10. Menen RS, Hassanein MK, Momiyama M, Suetsugu A, Moossa AR, Hoffman $\mathrm{RM}$, et al. Tumor-educated macrophages promote tumor growth and peritoneal metastasis in an orthotopic nude mouse model of human pancreatic cancer. In Vivo. 2012; 26: 565-569.

11. Shuman Moss LA, Jensen-Taubman S, Stetler-Stevenson WG. Matrix metalloproteinases: changing roles in tumor progression and metastasis. Am J Pathol. 2012; 181: 1895-1899. doi: 10.1016/j.ajpath.2012.08.044.

12. Jaguin M, Houlbert N, Fardel O, Lecureur V. Polarization profiles of human M-CSF-generated macrophages and comparison of M1-markers in classically activated macrophages from GM-CSF and M-CSF origin. Cell Immunol. 2013; 281: 51-61. doi: 10.1016/j.cellimm.2013.01.010.

13. Hamilton JA. Colony-stimulating factors in inflammation and autoimmunity. Nature Rev Immunol. 2008; 8: 533-544. doi: 10.1038/nri2356.

14. Fleetwood AJ, Lawrence T, Hamilton JA, Cook AD. Granulocyte-macrophage colony-stimulating factor (CSF) and macrophage CSF-dependent macrophage phenotypes display differences in cytokine profiles and transcription factor activities: implications for CSF blockade in inflammation. J Immunol. 2007; 178: 5245-5252.

15. Baay M, Brouwer A, Pauwels P, Peeters M, Lardon F. Tumor cells and tumor-associated macrophages: secreted proteins as potential targets for therapy. Clin Dev Immunol. 2011; 2011: 565187. doi: 10.1155/2011/565187.

16. Rebouissou S, Amessou M, Couchy G, Poussin K, Imbeaud S, Pilati C, et al. Frequent in-frame somatic deletions activate gp130 in inflammatory hepatocellular tumours. Nature. 2009; 457: 200-204. doi: 10.1038/nature07475.

17. Bode JG, Ehlting C, Haussinger D. The macrophage response towards LPS and its control through the p38(MAPK)-STAT3 axis. Cell Signal. 2012; 24: 1185-1194. doi: 10.1016/j.cellsig.2012.01.018.

18. Van Dyken SJ, Locksley RM. Interleukin-4- and interleukin-13-mediated alternatively activated macrophages: roles in homeostasis and disease. Annu Rev Immunol. 2013; 31: 317-343. 10.1146/annurev-immunol-032712-095906.

19. Shen Z, Kauttu T, Seppanen H, Vainionpaa S, Ye Y, Wang S, et al. Both macrophages and hypoxia play critical role in regulating invasion of gastric cancer in vitro. Acta Oncol. 2013; 52: 852-860. doi: 10.3109/0284186X.2012.718444.

20. Rey-Giraud F, Hafner M, Ries CH. In vitro generation of monocyte-derived macrophages under serum-free conditions improves their tumor promoting functions. PLoS ONE. 2012; 7: e42656. doi: 10.1371/journal.pone.0042656.

21. Helm O, Held-Feindt J, Grage-Griebenow E, Reiling N, Ungefroren H, Vogel I, et al. Tumor-associated macrophages exhibit pro- and anti-inflammatory properties by which they impact on pancreatic tumorigenesis. Int J Cancer. 2014; 135: 843-861. doi: 10.1002/ijc.28736.

22. Lewis CE, Pollard JW. Distinct role of macrophages in different tumor microenvironments. Cancer Res. 2006; 66: 605-612.

23. Dinarello CA. Why not treat human cancer with interleukin-1 blockade? Cancer Metastasis Rev. 2010;29: 317-329. doi: 10.1007/s10555-010-9229-0.

24. Balkwill F. Tumour necrosis factor and cancer. Nat Rev Cancer. 2009; 9: 361-371. doi: $10.1038 /$ nrc2628.

25. Friess H, Guo XZ, Nan BC, Kleeff J, Buchler MW. Growth factors and cytokines in pancreatic carcinogenesis. Ann N Y Acad Sci. 1999; 880: 110-121.

26. Qu N, Xu M, Mizoguchi I, Furusawa J, Kaneko K, Watanabe K, et al. Pivotal roles of T-helper 17-related cytokines, IL-17, IL-22, and IL-23, in inflammatory diseases. Clin Dev Immunol. 2013; 2013: 968549. doi: 10.1155/2013/968549.

27. Li J, Lau G, Chen L, Yuan YF, Huang J, Luk JM, et al. Interleukin 23 promotes hepatocellular carcinoma metastasis via NF-kappa B induced matrix metalloproteinase 9 expression. PLoS ONE. 2012; 7: e46264. doi: 10.1371/journal.pone.0046264.

28. Aldinucci D, Colombatti A. The inflammatory chemokine CCL5 and cancer progression. Mediators Inflamm. 2014; 2014: 292376. doi: $10.1155 / 2014 / 292376$

29. Hanahan D, Coussens LM. Accessories to the crime: functions of cells recruited to the tumor microenvironment. Cancer Cell. 2012; 21: 309-322. doi: 10.1016/j.ccr.2012.02.022.

30. Teng MW, Vesely MD, Duret H, McLaughlin N, Towne JE, Schreiber RD, et al. Opposing roles for IL-23 and IL-12 in maintaining occult cancer in an equilibrium state. Cancer Res. 2012; 72: 3987-3996. doi: 10.1158/0008-5472.CAN-12-1337.

31. Shen Z, Seppanen $H$, Kauttu $T$, Vainionpaa S, Ye Y, Wang S, et al Vasohibin-1 expression is regulated by transforming growth factor-beta/bone morphogenic protein signaling pathway between tumor-associated macrophages and pancreatic cancer cells. J Interferon Cytokine Res. 2013; 33: 428-433. doi: 10.1089/jir.2012.0046. 